\title{
Preliminary design and optimization of a G-band EIO based on pseudospark-sourced electron beam
}

\author{
Y. Yin ${ }^{1,2, a)}$, W. $\mathrm{He}^{2)}$, L. Zhang ${ }^{2)}, \mathrm{H}$. Yin $^{2)}$, and A. W. Cross ${ }^{2)}$ \\ ${ }^{1}$ School of Physical Electronics, University of Electronic Science and Technology of China, Chengdu, 610054, China \\ ${ }^{2}$ Department of Physics, Scottish Universities Physics Alliance, University of Strathclyde, Glasgow, G4 ONG, United \\ Kingdom
}

The design and simulation of a G-band extended interaction oscillator (EIO) driven by pseudospark-sourced electron beam is presented. The characteristic of the EIO and the pseudospark-based electron beam are studied to enhance the performance of the newly proposed device. The beam-wave interaction of the EIO can be optimized by choosing a suitable pseudospark discharging voltage and by widening the operating voltage region of the EIO circuit. Simulation results show that a peak power over $240 \mathrm{~W}$ can be achieved at G-band using a pseudospark discharging voltage of $41 \mathrm{kV}$.

\section{INTRODUCTION}

High frequency sources above $100 \mathrm{GHz}$ are very attractive for a wide range of research and technical applications $[1,2]$, including molecular spectroscopy, bio-imaging, security screening and communications. Vacuum electron devices (VEDs) [3], such as a backward-wave oscillator (BWO) can operate up to $1 \mathrm{THz}$ with milliwatt output powers. The gyrotron can generate kilowatt output power up to $1.0 \mathrm{THz}$ [4] when a high magnetic field is used. Free-electron lasers (FELs) can generate even higher pulse power at higher frequency bands than gyrotrons. However, compact and affordable sources above 100GHz with an intermediate power level of hundreds watts are in demand for a number of important applications. For example, dynamic nuclear polarization in high-field nuclear magnetic resonance spectroscopy requires radiation with a power of 10$100 \mathrm{~W}$ at frequencies such as 260,390 and $460 \mathrm{GHz}$. The extended interaction devices such as the extended interaction klystron (EIK) and extended interaction oscillator (EIO) has been widely studied $[5,6,7]$ as promising sources to satisfy this application as they combine the ruggedness and high power capability of a klystron and the wide bandwidth of a travelling wave tube. To a significant extent, the power limit from the EIK/EIO above $100 \mathrm{GHz}$ is a consequence of the difficulty in producing small, high current density, precisely-aligned electron beams [8]. To transport a high current density electron beam in a small beam tunnel, a large external magnetic field is usually used $[9,10]$. However, recent study on the pseudosparksourced electron beam demonstrated that electron beam pulses of thousands Amperes can be transported in a 3 mm diameter tunnel without a guiding magnetic field [11]. The pseudospark-sourced electron beam enables a new way to generate millimeter and terahertz waves to be achieved.

a) Electronic mail: yong.yin@strath.ac.uk yinyong@uestc.edu.cn 
This paper presents the design and simulation of a G-band (140-220GHz) EIO based on a pseudospark-sourced electron beam. The characteristics of the pseudospark-based electron beam is studied to optimize the performance of the G-band EIO. In Section II, a brief introduction to the characteristic of the pseudospark-source electron beam is given. Section III includes the design of the G-band EIO. In Section IV, influence of the pseudospark-based electron beam characteristics on the performance of the EIO is discussed. Conclusions are contained in Section V.

\section{CHARACTERISTIC OF THE PSEUDOSPARK-SOURCED ELECTRON BEAM}

A pseudospark discharge is an axially symmetric, self-sustained, transient, low pressure (typically 50-500 mtorr) gas discharge in a hollow cathode and planar anode configuration and operates on the left-hand side (with respect to the minimum) of the Paschen curve. A typical four-gap pseudospark discharge setup is shown in Fig.1 [12]. The hollow cathode (pseudospark chamber) was connected to the DC power supply through a $30 \mathrm{M} \Omega$ charging resistor. The anode was grounded and there was no external applied guide magnetic field. The electron beam was extracted from a four-gap pseudospark discharge chamber.

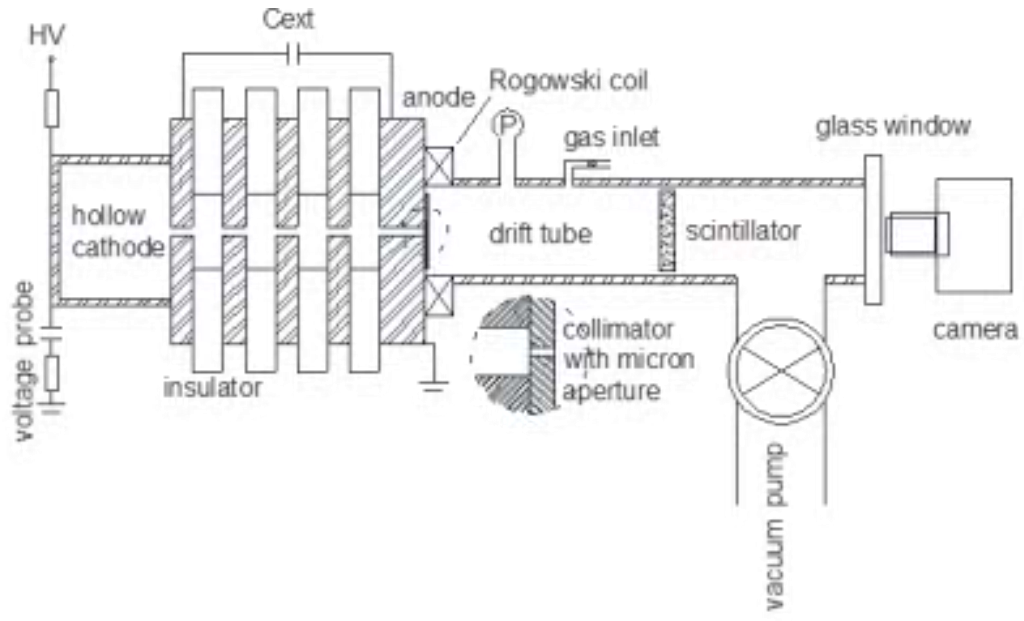

FIG. 1. A typical pseudospark-sourced electron beam experimental setup.

A $2.5 \mathrm{~A}, 50 \mathrm{kV}$ electron beam pulse was measured at a distance of $20 \mathrm{~mm}$ from the acceleration gap without a guide magnetic field, as shown in Fig.2. At the start of the pseudospark discharge the initial high-energy electrons ionizes the background gas in the drift tube so that a plasma channel could be formed. The following beam electrons generated by the pseudospark discharge then expel part of the plasma electrons in the channel due to expulsive electric force while the much more massive ions remain relatively fixed. The resulting positive ion channel acts to focus and guide the pseudosparksourced electron beam. The shape and the size of the beam can be formed by fabricating a required aperture in the collimator.

\section{[Type text]}




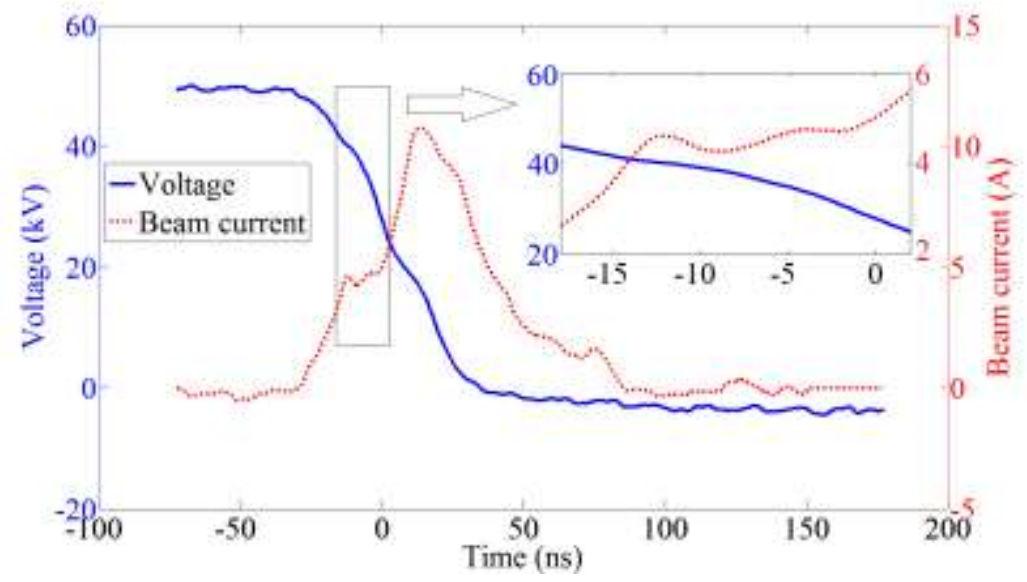

FIG. 2 A temporal evolution of the electron beam produced from a four-gap pseudospark discharge.

The generated electrons have the variable voltage and current, but there exists a hollow cathode discharge period when the current and voltage stayed almost unchanged in about 10 to $15 \mathrm{~ns}$. The stable current and voltage region could be used to drive the EIO. The duration of this relatively stable period can be varied through adjusting the capacitance of external capacitor $\mathrm{C}_{\mathrm{ext}}$. Typically for the full discharging process the voltage is changing from the applied voltage to almost zero in about $30-100 \mathrm{~ns}$, i.e. with a voltage change rate of approximately $1 \mathrm{kV} / \mathrm{ns}$ and a current density over $1 \mathrm{kA} / \mathrm{cm}^{2}$.

\section{DESIGN OF THE G-BAND EIO}

Fig.3 shows the schematic drawing of a nine-slot EIO circuit operating in the G-band (a) and the dispersion curve (b). The number of the slots is optimized to obtain efficient circuit impedance. The red cylinder is the electron beam tunnel which intersects with the slow-wave structure consisting of several small rectangular gaps. The up and down rectangular section forms the extended resonant cavity of the EIO circuit. The velocity of the electron beam (or the beam energy) is chosen so that the time interval between the electron travels from one slot to the adjacent one is equal to the period of the electronmagnetic wave supported by the slow wave structure, thereby ensuring an efficient interaction between the beam and the interaction circuit. The operation mode is often determined by the electric field variation along the electron beam tunnel in one period.

The synchronous condition of the EIO is

$$
\frac{P}{v_{e}}=N T=\frac{N}{f}
$$

Where $P$ is the period of the slots, $v_{e}$ is the velocity of the electron beam, $T$ and $f$ is the period and frequency of the electromagnetic wave supported by the interaction circuit, respectively. $N$ is a constant related to the operation mode in the slow wave structure. When the operation mode in the slow wave structure is a $2 \pi$ mode, the phase difference between two [ 1 y Y 
adjacent slots is $2 \pi$. The synchronous condition implies that the time interval between the electrons traveling from one slot to the next one is equal to the period of the electromagnetic wave supported by the interaction circuit resulting in $N$ equal to 1 . In another situation, when $N$ equals to 0.5 the operation mode is $\pi$, and so on. The $2 \pi$ mode is chosen as the operation mode, because it has a large period of the circuit under the same operation voltage and frequency. The extended interaction circuit has a number of benefits, primarily raising the coupling impedance of the circuit enabling efficient modulation of the electron beam across a broad frequency band. Distributing the electric field across several gaps within a single cavity also has the benefit of reducing the electric field in the gaps, reducing the chances of breakdown and arcing. The high impedance of the extended interaction circuit reduces the total length of the interaction circuit, thus ensuring beam transmission.

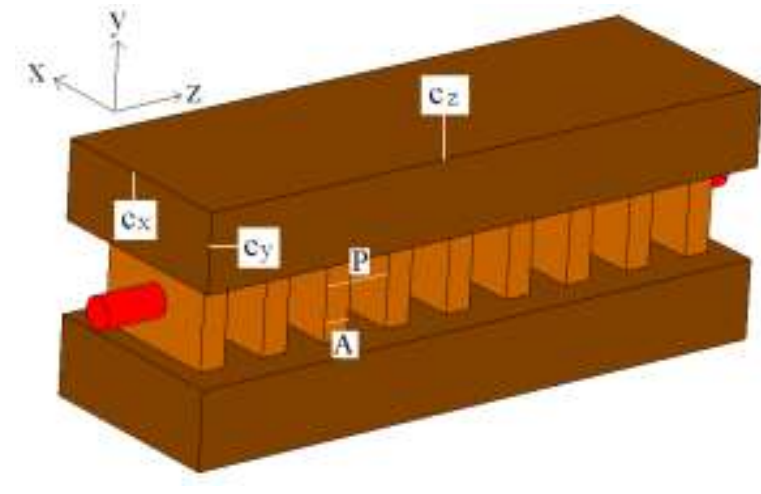

(a)

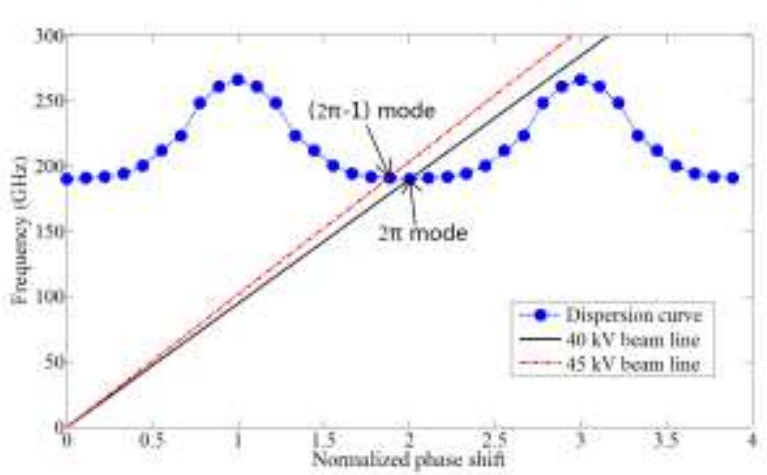

(b)

FIG. 3 Schematic drawing of a G-band EIO circuit with nine-slot (a) and the dispersion curve (b).

Once the operation frequency and mode is decided, the relation between the period and the electron beam velocity (hence the operation voltage) can be obtained from equation (1). For a $40 \mathrm{kV}$ electron beam, the required period for $2 \pi$ mode operation is $0.56 \mathrm{~mm}$. The depth and width of the slots is chosen to get the desired circuit frequency and appropriate circuit impedance which dictates the size of the cavity as follows. Firstly, the cavity width $C_{x}$ is fixed to $1.30 \mathrm{~mm}$ for G-band output because this is the width of WR5 waveguide operating in G-band. Secondly, the cavity height $C_{y}$ is chosen to ensure the field $E_{z}$ satisfied the boundary conditions at $y$ maximum and minimum which is related to the operation frequency of the circuit with a final size of $0.3 \mathrm{~mm}$. Finally a cavity length $C_{z}$ of $5.0 \mathrm{~mm}$ was chosen to satisfy the magnetic field boundary conditions at the metallic surface and to have the half-wave number equal to the slot separation.

For the above conditions the $Q_{0}$ and the operation frequency is determined, the output structure is also an important aspect in designing the device. The load of this device is mainly having two parts, one due to the surface loss in the circuit and the other is the power extracted from the system therefore equation (2) exists.

\section{[Type text]}




$$
\frac{1}{Q_{l}}=\frac{1}{Q_{0}}+\frac{1}{Q_{e}}
$$

here $Q_{0}$ and $Q_{e}$ is the intrinsic and external quality factors of the extended interaction circuit, $Q_{l}$ is the loaded quality factor.

In practice, the surface loss on copper may vary with the surface roughness. The non-ideal surface losses at high frequencies caused by surface roughness has been reported [13,14], the following formula is used to estimate the effective conductivity as a function of surface roughness.

$$
\sigma_{\text {eff }}=\frac{\sigma_{\text {ide }}}{\left(1+\exp \left(-\left(\frac{\delta_{\text {ide }}}{2 h}\right)^{1.6}\right)\right)^{2}}
$$

Here, $\sigma_{i d e}$ is the conductivity of the metals having an ideal surface, $\delta_{i d e}$ is the skin depth for the ideal metals with smooth surface and $h$ is the surface roughness measured as the RMS deviation of the surface from its mean planar position. The skin depth of the copper at $200 \mathrm{GHz}$ is about $0.15 \mu \mathrm{m}$.

At $200 \mathrm{GHz}$, the $Q_{0}$ is difficult to achieve for very high values for the high frequency loss of the copper is quite large. For example a surface roughness of $0.075 \mu \mathrm{m}$ (half the ideal skin depth) will double the surface loss compared to the ideal case. In our design, the $Q_{e}$ was chosen to be larger than the $Q_{0}$ to ensure a short start oscillation time, which is critical for the device when driven by the pseudospark-sourced electron beam. And the $Q_{e}$ can be varied through change in the radius of the coupling hole.

Interaction between the electron beam and the EIO interaction circuit was studied. A DC beam with 40keV energy which satisfied the synchronous condition of the EIO, was injected into the interaction circuit. A particle-in-cell code MAGIC [15] is used to simulate the beam-wave interaction process. The beam diameter is $0.28 \mathrm{~mm}$, the related current density is $500 \mathrm{~A} / \mathrm{cm}^{2}$. The interaction circuit is a nine-slot slow wave structure with two cavities. When the synchronous condition of the equation (1) is satisfied, the oscillation can occur and the power can be extracted out from the output structure. Fig. 4 is the phase space plot of electrons at Z-Y (a) and Z-Pz (b). Because the interaction between the circuit and the electrons, some electrons lose the energy to the electromagnetic field.

\section{[Type text]}




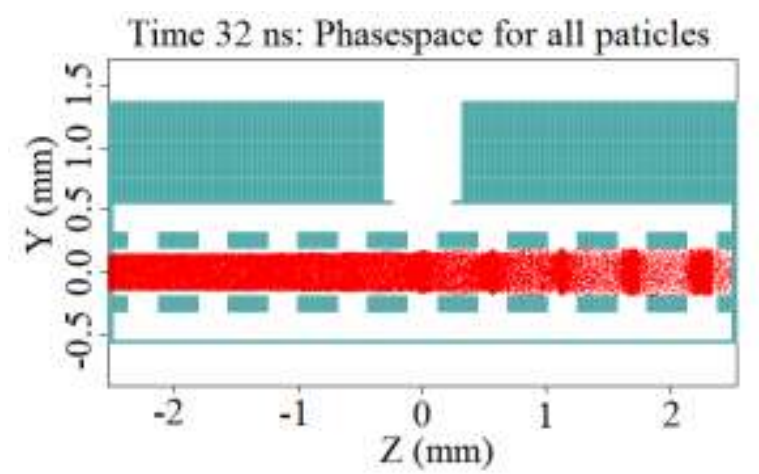

(a)

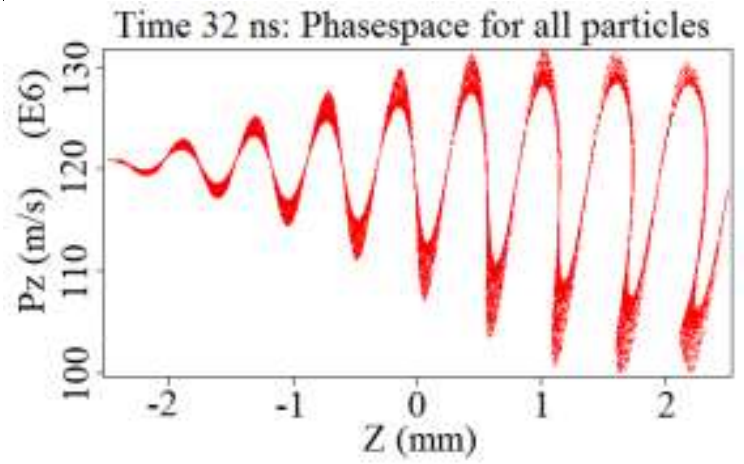

(b)

FIG. 4. The phase space plots of electrons at Z-Y (a) and Z-Pz (b).

Fig. 5(a) gives the output power as determined by integration of the Poynting flux across the output waveguide. Fig. 5(b) is the frequency spectrum of a point's angular electric field in the output structure. The average output power is $330 \mathrm{~W}$ and the main frequency is at $188.8 \mathrm{GHz}$. The field contours of $\mathrm{E}_{\mathrm{z}}$ in the circuit are plotted in Fig. 6 when the oscillation in the circuit is established.

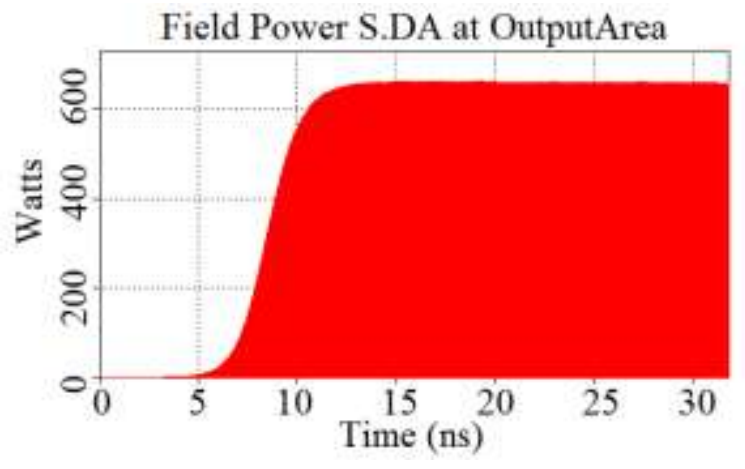

(a)

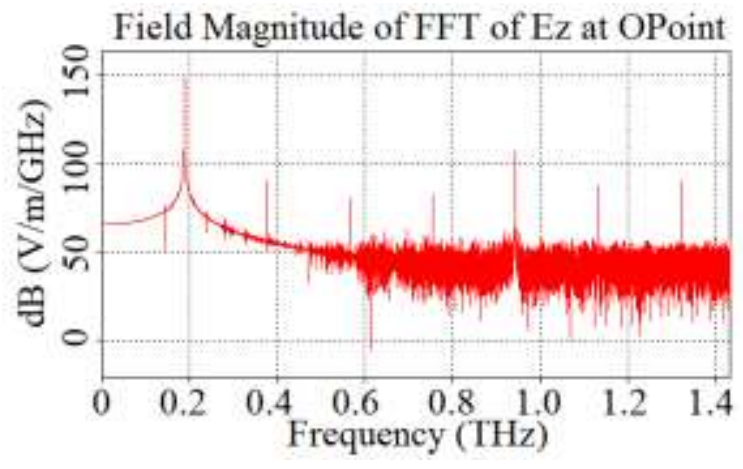

(b)

FIG. 5 Time plot of the output power (a) and the frequency spectrum of a point's $\mathrm{E}_{\mathrm{z}}$ in output structure (b).

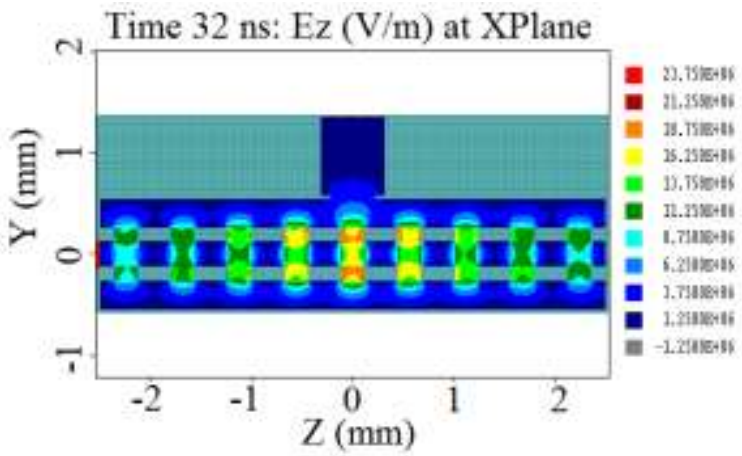

(a)

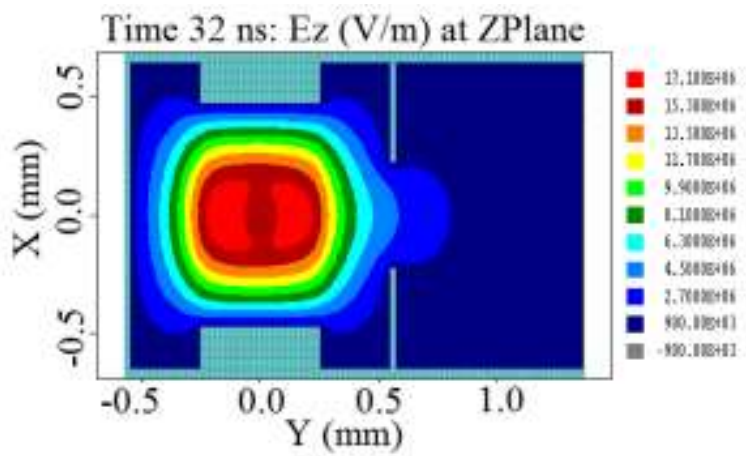

(b)

FIG. 6. Oscillated field contours of $E_{z}$ in the circuit at Z-Y (a) and Y-X (b) cut plane.

\section{EFFECTS OF THE CHARACTERISTICS OF PSEUDOSPARK-BASED ELECTRON BEAM ON THE PERFORMANCE OF THE EIO}

[Type text] 
As mentioned in the previous sections, the operating voltage region and the oscillation startup time are important features of the EIO for the pseudopark-sourced electron beam. In the EIO, the electron beam interacts with the standing wave (zero group velocity) of the circuit. Fig. 7 shows the simulated effects of the beam voltages on the output power and oscillation startup time. The fast falling edges of the output power at $44 \mathrm{kV}$ is due to the change of the operating mode from $2 \pi$ to $2 \pi-1$ mode, as shown in Fig. 3(b). When operated in $2 \pi-1$ mode, the $Q_{0}$ will decrease and the $Q_{e}$ will increase, this lowers the efficiency of the interaction and also the output power dramatically. The operating voltage region of the $2 \pi$ mode EIO is 7 $\mathrm{kV}$. At the designed operation voltage $(40 \mathrm{kV})$, the minimum oscillation startup time of this device is about $7.3 \mathrm{~ns}$.

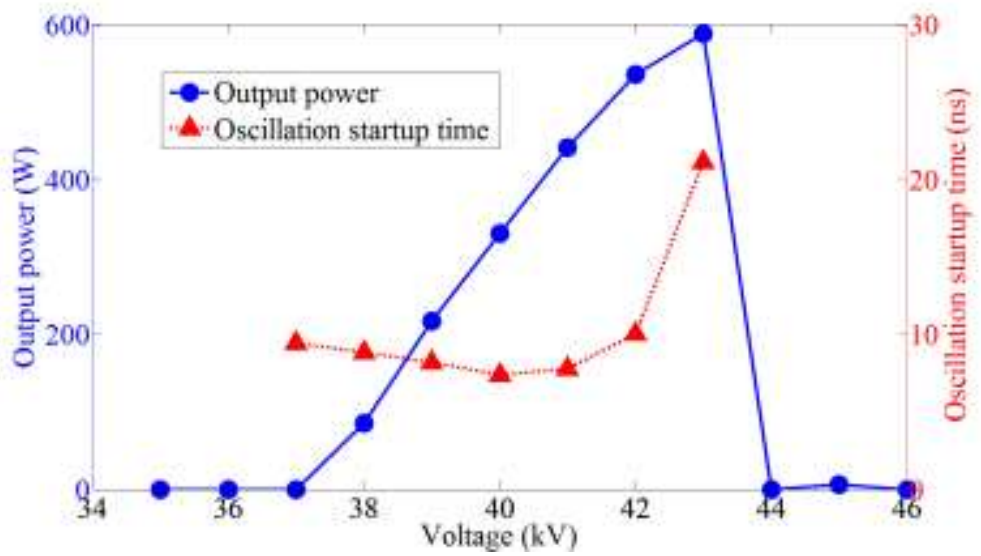

FIG. 7 The effects of the beam voltage on the output power and oscillation startup time.

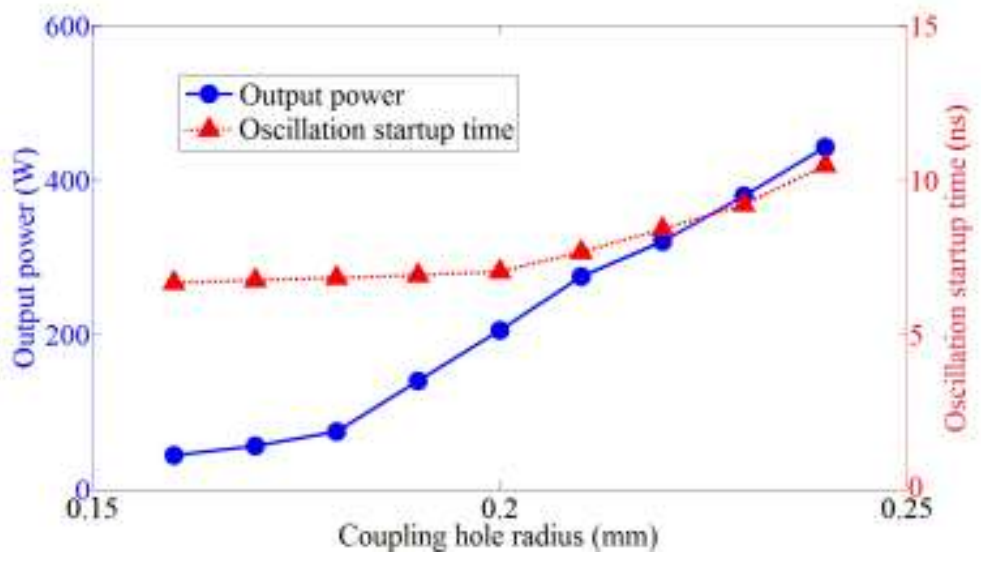

FIG. 8 The effects of the radius of the coupling hole on the output power and oscillation startup time. 


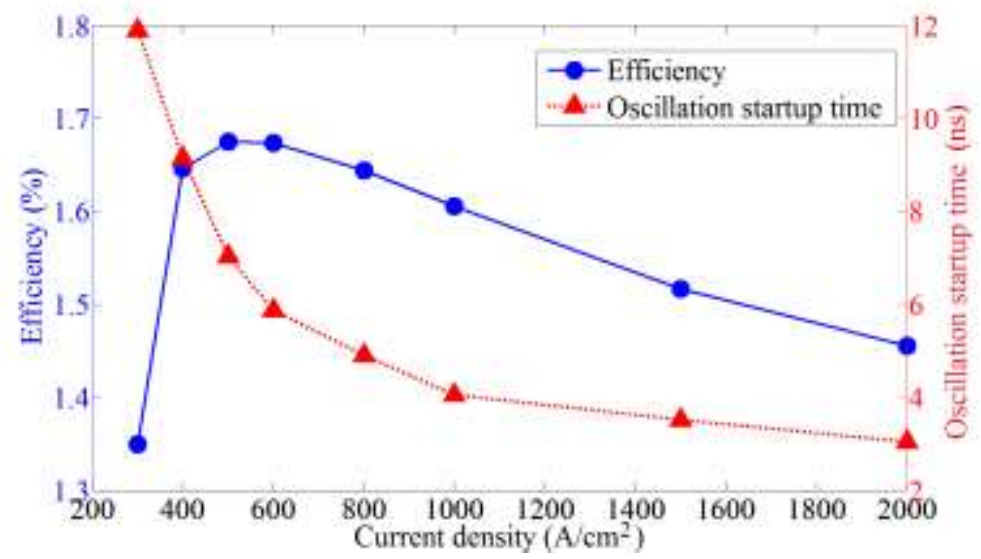

FIG. 9 The effects of the current density to the efficiency and oscillation startup time.

Fig. 8 shows the simulated effects of the radius of coupling hole on the output power and oscillation startup time. The smaller the coupling hole is, the higher the external quality factor $Q_{e}$. A smaller $Q_{e}$ will give rise to a shorter oscillation startup time and a reduced output power. A good choice for the radius of the coupling hole is $0.2 \mathrm{~mm}$, correspondingly the oscillation startup time is $7.0 \mathrm{~ns}$. The effects of the current density on the interaction efficiency and oscillation startup time were also simulated and are shown in Fig. 9. With the increase of the current density, and hence the beam current, the output power would increase to a saturation point and then decrease. The oscillation startup time would decrease with the increase of beam current. But when the beam current increases to a certain value, the decrease of the oscillation startup time is limited. This might be a result from the detrimental effect of space charge on the formation of bunching [16]. If the current density is more than $500 \mathrm{~A} / \mathrm{cm}^{2}$, the oscillation startup time is predicted to be smaller than $7.0 \mathrm{~ns}$.

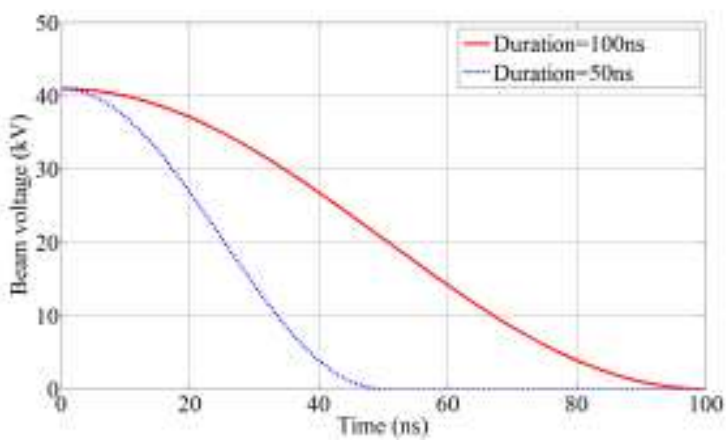

(a)

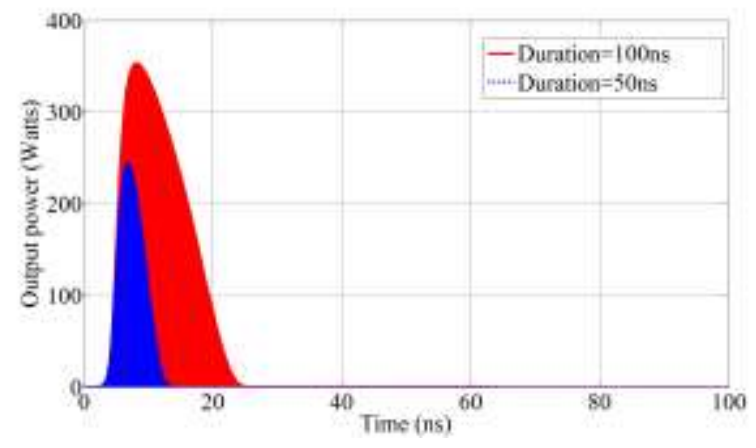

(b)

FIG. 10 The electron beam voltage (a) and output power (b) as a function of time.

In the PIC simulation, an electron beam with time varied voltage (as shown in Fig.10 (a)) with a constant $300 \mathrm{~mA}$ current was injected in the nine-slot extended interaction circuit. When the voltage of pseudospark beam was changed from $41 \mathrm{kV}$ to $0 \mathrm{kV}$ in $50 \mathrm{~ns}$, an output power of $240 \mathrm{~W}$ with duration of $4-13 \mathrm{~ns}$ is predicted from simulation and is shown in Fig. 10 (b) by the doted blue line. In the time interval, the beam voltage was $37-44 \mathrm{kV}$ which is in the designed working voltage 
of the EIO. Fig. 11 (a) shows the beam voltage change from $50 \mathrm{kV}$ to $0 \mathrm{kV}$ in $50 \mathrm{~ns}$. Fig. 11 (b) shows the output power has two regions. The first region (6-12ns) of $4 \mathrm{~W}$ power is related to a higher voltage region (44-48 $\mathrm{kV})$, which is operated in the $2 \pi-1$ mode in the extended interaction circuit. Because the output structure is designed for $2 \pi$ mode operation, the output power is significant lower than the designed operating mode, at a center frequency of $190.3 \mathrm{GHz}$. By the use of a lower discharge voltage, this interaction can be avoided. The output power of the second region (15-18ns) of $36 \mathrm{~W}$ power is related to the designed voltage region, which is operated in the $2 \pi$ mode.

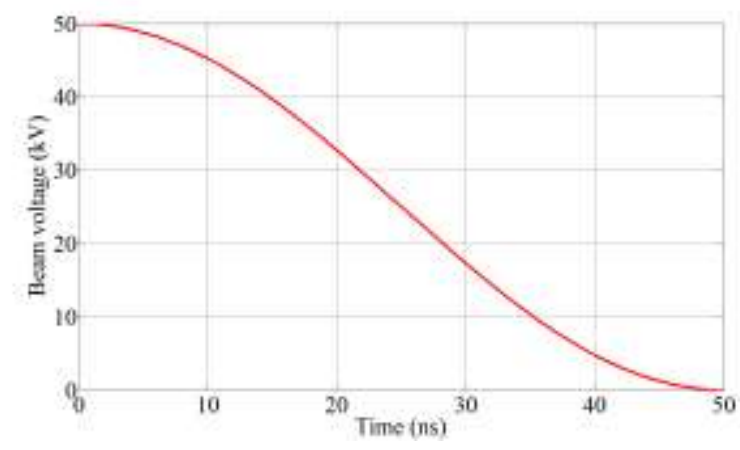

(a)

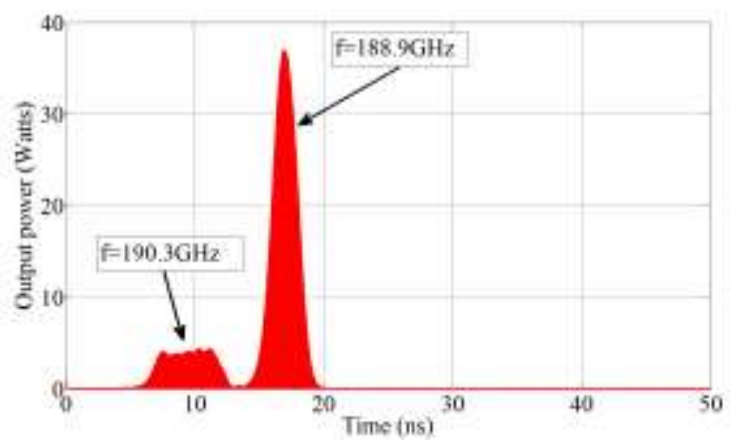

(b)

FIG. 11. The electron beam voltage (a) and output power (b) as a function of time.

The reason for the difference between Fig.10(b) and Fig.11(b) is the beam voltage duration because the EIO can only oscillate in a limited voltage region, hence the more time the beam voltage is in this region, the more output power can be obtained. As for the pseudospark-sourced electron beam, a lower rate of change in the beam voltage can ensure more electrons are located in the oscillating voltage region over a relatively longer time, thus improving the output power of the device. In pseudospark discharge, the discharging duration could be adjusted by applying different external capacitances. If the voltage reduces from $41 \mathrm{kV}$ to 0 in $100 \mathrm{~ns}$, and the other parameters kept the same as used in Fig. 10, an output power of $345 \mathrm{~W}$ with duration of 4-22 ns is predicted from simulation as shown in Fig. 10 (b) by the solid red line. For the device itself, if the EIO circuit has a wide operation voltage region, this will also benefit the performance of the device, especially when driven by a pseudospark-sourced electron.

\section{DISCUSSION AND CONCLUSION}

This paper combines of the merit of a short interaction circuit in the EIO and the high current density property of the pseudospark-sourced electron beam to generate electromagnetic radiation in G-band. The design and optimization of the Gband EIO with a pseudospark-sourced electron beam has been carried out. Simulation results show that a peak power over 240W can be achieved at G-band using a pseudospark discharging voltage of $41 \mathrm{kV}$. The effect of a voltage change rate of 
about $1 \mathrm{kV} / \mathrm{ns}$ for the same beam current on EIO performance was also studied. The key features in designing the pseudospark-sourced EIO is a stable and wide operating voltage region.

It should be mentioned that this study of EIO based on the pseudospark-sourced electron beam has neglected the effects of the plasma in the performance of the EIO. The unmagetized plasma, can be considered as a dielectric media with a dielectric constant of $\varepsilon_{r}=1-\omega_{p e}{ }^{2} / \omega^{2}$. As the designed operating frequency is far away from the plasma frequency the plasma would have negligible effect on the output power of the EIO. However the plasma would upshift the output frequency slightly, and from the dispersion equation, it is estimated to be approximately $10 \%$. Our next step is to design and manufacture a G-band EIO circuit with a wide operating voltage region, and to utilize a large external capacitor in the pseudospark-sourced electron beam experimental setup to drive this circuit. This shows a new way to generate several hundred watts of power in the millimeter and even terahertz frequency range from a pseudospark-sourced electron beam in a compact and affordable way.

\section{ACKNOWLEDGMENTS}

This work was supported by the National Natural Science Foundation of China (Grant No. 61201011), the Fundamental Research Funds for the Central Universities (Grant No. ZYGX2013J056), and the Oversea Academic Training Fund, University of Electronic Science and Technology of China and EPSRC, UK (research grant EP/G011087/1).

\section{REFERENCES}

${ }^{1}$ P. H. Siegel, IEEE Trans. on Microwave Theory and Techniques, 50, 910 (2002).

${ }^{2}$ P. H. Siegel, IEEE Trans. on Microwave Theory and Techniques, 52, 2438 (2004).

${ }^{3}$ J. H. Booske, R. J. Dobbs, C. D. Joye, C. L. Kory, G. R. Neil, G. S. Park, J. Park, R. J. Temkin, IEEE Trans. on Terahertz Science and Technology, 1, 54 (2011).

${ }^{4}$ M. Yu. Glyavin, A. G. Luchinin and G. Yu. Golubiatnikov, Phy. Rev. Lett., 100, 015101 (2008).

${ }^{5}$ Roitman, D. Berry and B. Steer, IEEE Trans. on Electron Devices, 52, 895 (2005).

${ }^{6}$ M. Chodorow, B. Kulke, IEEE Trans. on Electron Devices, ED-13, 439 (1966).

${ }^{7}$ Y. M. Shin, G. S. Park, G. P. Scheitrum, G. Caryotakis, Journal of the Korean Physical Society, 44, 1239 (2004).

${ }^{8}$ J. H. Booske, Physics of Plasmas, 15, 055502 (2008).

${ }^{9}$ K. T. Nguyen, J. A. Pasour, T. M. Antonsen, P. B. Larsen, J. J. Petillo, B. Levush, IEEE Trans. on Electron Devices, 56, 744 (2009).

[Iype text] 
${ }^{10}$ J. H. Booske, M. A. Basten, A. H. Kumbasar, T. M. Antonsen Jr., S. W. Bidwell, Y. Carmel, W. W. Destler, V. L. Granatstein, D. J. Radack, Physics of Plasmas, 1, 1714 (1994).

${ }^{11}$ H. Yin , A. W. Cross, A. D. R. Phelps, D. Zhu , W. He, K. Ronald, Journal of Applied Physics 91, 5419 (2002).

${ }^{12}$ H. Yin, A. W. Cross, W. He, A. D. R. Phelps, K. Ronald, D. Bowes, C. W. Robertson, Physics of Plasmas, 16, 063105, (2009).

${ }^{13}$ S. K. Datta, L. Kumar, B. N. Basu, Journal of Infrared, Millimeter and Terahertz Waves, 30, 381 (2009).

${ }^{14}$ M. P. Kirley, N. Carlsson, B. B. Yang, J. H. Booske, in Proc. 11th IEEE International Vacuum Electronics Conference, Monterey, April 24-26, 2012, p. 239-240.

${ }^{15}$ B. Goplen, L. Ludeking, D. Smithe, and G. Warren, Comput. Phys. Commun., 87, 54 (1995).

${ }^{16}$ T. G. Mihran, IRE Trans. on Electron Devices, 6, 54 (1959). 